\title{
Sex-dependent effect of a low neurosteroid environment and intrauterine growth restriction on foetal guinea pig brain development
}

\author{
Meredith A Kelleher, Hannah K Palliser, David W Walker ${ }^{1}$ and Jonathan J Hirst \\ Mothers and Babies Research Centre, John Hunter Hospital and School of Biomedical Sciences, University of Newcastle, Newcastle, New South Wales 2310, \\ Australia \\ ${ }^{1}$ Department of Physiology, Monash University, Clayton, Victoria 3168, Australia \\ (Correspondence should be addressed to M A Kelleher; Email: meredith.kelleher@uon.edu.au)
}

\begin{abstract}
Progesterone and its neuroactive metabolite, allopregnanolone, are present in high concentrations during pregnancy, but drop significantly following birth. Allopregnanolone influences foetal arousal and enhances cognitive and behavioural recovery following traumatic brain injury. Inhibition of allopregnanolone synthesis increases cell death in foetal animal brains with experimental hypoxia. We hypothesised that complications during pregnancy, such as early or preterm loss of placental steroids and intrauterine growth restriction (IUGR), would disrupt the foetal neurosteroid system, contributing to poor neurodevelopmental outcomes. This study aimed to investigate the effects of chronic inhibition of allopregnanolone synthesis before term and IUGR on developmental processes in the foetal brain. Guinea pig foetuses were experimentally growth restricted at midgestation and treated with finasteride, an inhibitor of allopregnanolone synthesis. Finasteride treatment reduced
\end{abstract}

foetal brain allopregnanolone concentrations by up to $75 \%$ and was associated with a reduction in myelin basic protein (MBP) $(P=0 \cdot 001)$ and an increase in glial fibrillary acidic protein expression in the subcortical white matter brain region $(P<0 \cdot 001)$. IUGR resulted in decreased $\mathrm{MBP}$ expression $(P<0 \cdot 01)$ and was associated with a reduction in the expression of steroidogenic enzyme $5 \alpha$-reductase $(5 \alpha \mathrm{R})$ type 2 in the foetal brain $(P=0 \cdot 061)$. Brain levels of $5 \alpha \mathrm{R} 1$ were higher in male foetuses $(P=0 \cdot 008)$. Both IUGR and reduced foetal brain concentrations of allopregnanolone were associated with altered expression of myelination and glial cell markers within the developing foetal brain. The potential role of neurosteroids in protecting and regulating neurodevelopmental processes in the foetal brain may provide new directions for treatment of neurodevelopmental disorders in infants who are exposed to perinatal insults and pathologies. Journal of Endocrinology (2011) 208, 301-309

\section{Introduction}

Foetuses that are born small for gestational age due to intrauterine growth restriction (IUGR) are at higher risk for perinatal morbidity, mortality and long-term disability (Larroque et al. 2001). Abnormal foetal growth is associated with a high risk of foetal brain injury, leading to postnatal motor disorders, neurodevelopmental delay and long-term cognitive impairments. The risk of preterm birth is also higher in infants who are growth restricted or small for gestational age (Lackman et al. 2001). Placental insufficiency and IUGR have many implications for foetal brain development. Along with clinical observations, animal studies have also revealed the morphological changes and neurological impairments associated with foetal IUGR. In foetal sheep, experimental chronic placental insufficiency late in gestation impairs neural development and results in white matter damage and a reduction in the density of pyramidal cells within the hippocampus (Rees et al. 1998). In pregnant guinea pigs, IUGR induced by experimentally limiting

uterine blood flow to the placenta results in reduced hippocampal volume and fewer hippocampal pyramidal neurons in the foetal brain at the end of gestation (Mallard et al. 2000).

Progesterone is present in high concentrations throughout pregnancy, and plasma concentrations increase in both the maternal and foetal circulations during late gestation in humans, nonhuman primates and guinea pigs (Gilbert Evans et al. 2005, Mitchell \& Taggart 2009). Levels of allopregnanolone, a major metabolite of progesterone, increase concurrently with progesterone over the course of gestation in both tissues and plasma (Gilbert Evans et al. 2005). In the brain, allopregnanolone has positive modulatory actions at the $\gamma$-aminobutyric acid type $\mathrm{A}\left(\mathrm{GABA}_{\mathrm{A}}\right)$ receptor, causing neuronal hyperpolarisation and inhibition of neural activity, resulting in anxiolytic (de Brito Faturi et al. 2006), sedative (Paul \& Purdy 1992) and anticonvulsant (Kokate et al. 1999) effects. Allopregnanolone has also been shown to influence foetal arousal and sleep-wake activity in utero (Nicol et al. 1997), demonstrating that synthesis and release of this steroid 
has a physiological and regulatory role within the central nervous system (CNS).

Allopregnanolone is converted from progesterone by the enzymes $5 \alpha$-reductase types 1 and $2(5 \alpha \mathrm{R} 1 ; 5 \alpha \mathrm{R} 2)$ and $3 \alpha$-hydroxysteroid oxidoreductase $(3 \alpha$-HSOR; Compagnone \& Mellon 2000). These enzymes are present in the adrenal gland, ovary, brain and placenta. De novo synthesis of allopregnanolone from cholesterol wholly within the CNS is also possible (Poletti et al. 1998).

Endogenous neurosteroids, such as allopregnanolone, have been implicated in the regulation of normal brain development and may be particularly important to the foetal brain exposed to suboptimal or adverse conditions in utero, including pregnancies complicated by placental insufficiency and IUGR. For example, in a foetal sheep model of chronic placental insufficiency, expression of $5 \alpha \mathrm{R} 2$ increased in many regions of the foetal brain following late gestation umbilicoplacental embolisation, a procedure that reduces the area of the placental vasculature available for nutrient exchange (Nguyen et al. 2003).

There is increasing evidence that allopregnanolone has a neuroprotective function in both the adult (Djebaili et al. 2004, He et al. 2004, Schumacher et al. 2007) and foetal brains (Yawno et al. 2007, 2009). Inhibition of allopregnanolone synthesis, using finasteride (a $5 \alpha \mathrm{R}$ inhibitor), in foetal sheep increased the number of cells undergoing programmed cell death within the brain, and accentuated the cell death caused by asphyxia arising from transient umbilical cord occlusion (Yawno et al. 2007). Inhibition of $5 \alpha \mathrm{R}$ also appears to influence cell proliferation in the foetal sheep brain, with an increase in proliferating astrocytes observed in the hippocampus and cerebellum after finasteride treatment (Yawno et al. 2009).

We have previously shown that $5 \alpha \mathrm{R} 1$ and $5 \alpha \mathrm{R} 2 \mathrm{mRNA}$ levels are altered within the foetal guinea pig brain in a sex-specific manner in response to uterine artery ablation, a procedure that produces IUGR in this species (McKendry et al. 2010). This disruption of neurosteroid synthesis in pregnancies with perturbed placental function may result in vulnerability to foetal brain injury. In human pregnancies, IUGR often occurs in association with preterm birth (Lackman et al. 2001), and prematurity is itself a risk factor for perinatal brain damage (Saigal \& Doyle 2008). It is also known that foetal sex affects susceptibility to brain injury, with perinatal brain injury occurring more frequently in male infants (Di Renzo et al. 2007). A reduction in foetal allopregnanolone concentrations in late gestation may mimic the removal of progesterone and neurosteroid support as occurs at birth, either at term or with preterm delivery. We hypothesised that complications during pregnancy, such as IUGR, would disrupt the foetal neurosteroid system, contributing to poor neurodevelopmental outcomes in foetuses with these pregnancy complications. This study aimed to investigate the effects of IUGR, the chronic inhibition of allopregnanolone synthesis and the combination of these insults on late developmental processes within the foetal brain.

\section{Materials and Methods}

\section{Animals}

All animal procedures were approved by the University of Newcastle Animal Care and ethics committee and carried out in accordance with the Australian Code of Practice for the Care and Use of Animals for Scientific Purposes. Time-mated outbred tricolour guinea pigs, supplied by the University of Newcastle Research Support Unit, were housed indoors under a $12 \mathrm{~h}$ light: $12 \mathrm{~h}$ darkness cycle. Animals were provided with commercial guinea pig pellets and water fortified with ascorbic acid was made available ad libitum.

Surgery was performed under strict aseptic conditions between 33 and 35 days gestation to induce placental insufficiency and foetal IUGR. Food was removed $4 \mathrm{~h}$ prior to the surgery and the pregnant guinea pig dams then received $0.5 \mathrm{mg} / \mathrm{kg}$ Temgesic (324 $\mathrm{g}$ buprenorphine $\mathrm{HCl}$ and glucose anhydrous/ml, Reckitt Benckiser Healthcare Ltd, Hull, UK) prior to induction of general anaesthesia with $4 \%$ isoflurane in oxygen and maintenance of anaesthesia with $2 \%$ isoflurane in oxygen. A midline abdominal incision was made, the uterus was exposed and $\sim 50 \%$ of the radial arteries supplying each placenta were ablated using diathermy (Turner \& Trudinger 2009). The uterus was then replaced and the incision closed. Animals were returned to individual cages and received a second dose of Temgesic at $8 \mathrm{~h}$ post surgery. A control group of animals received sham surgery in which the same procedures were followed but no ablation of radial arteries was performed.

Animals were divided into groups based on surgery (sham/normally grown or IUGR) and daily drug treatment (vehicle or finasteride). This resulted in four foetal groups: control (sham, vehicle treated), IUGR (growth restricted, vehicle treated), FIN (sham, finasteride treated) and IUGR + FIN (growth restricted, finasteride treated). The pregnant dams received daily s.c. injections of vehicle $(600 \mu \mathrm{l} / \mathrm{kg} ; 16 \%$ $\mathrm{v} / \mathrm{v}$ ethanol in peanut oil) or finasteride $(25 \mathrm{mg} / \mathrm{kg}$; Steraloids, New York, NY, USA) from day 55 until they were killed at 65 days of gestation by carbon dioxide inhalation. Term in this colony of guinea pigs is $71 \pm 0.5$ days.

\section{Tissue collection}

Foetuses were removed from the uterus and foetal sex, body weight and organ weights were recorded. The foetal brain was removed from the skull within 2-3 min of maternal death before being weighed, hemisected and divided coronally into rostral, middle and caudal blocks. Blocks from the right hemisphere were snap frozen in liquid nitrogen and stored at $-80{ }^{\circ} \mathrm{C}$ before being finely crushed for protein and steroid extraction. Tissues from the left hemisphere were fixed by immersion in paraformaldehyde (PFA) (4\% w/v PFA in $0 \cdot 1 \mathrm{M}$ phosphate buffer). The blocks used in this study (middle block) contained the cerebral cortex, subcortical white matter, corpus callosum, thalamus and hippocampus. 


\section{RIA}

Allopregnanolone was extracted from brain tissue and measured by RIA using previously described methods (McKendry et al. 2010). Briefly, crushed frozen brain tissue was treated with $50 \%$ methanol with $1 \%$ acetic acid and added to Sep-Pak $\mathrm{C}_{18}$ cartridges (Waters, Milford, MA, USA) for separation of steroid components using methanol gradients. Residual methanol was removed by vacuum drying. In order to minimise cross-reactivity, the concentration of progesterone was reduced by treating the samples with potassium permanganate to oxidise non-saturated steroids. Recovery was measured by the addition of tritium-labelled allopregnanolone (1000-1500 c.p.m., 5 $\alpha-\left[9,11,12,{ }^{3} \mathrm{H}(\mathrm{N})\right]$; PerkinElmer Life and Analytical Sciences, Boston, MA, USA) to each sample prior to extraction. Each sample was corrected for its extraction loss in the final calculation of allopregnanolone concentrations. The average recovery was $57 \cdot 8 \pm 2 \cdot 6 \%$. Allopregnanolone was quantified by RIA using a polyclonal antibody supplied by Dr R H Purdy (Department of Psychiatry, Veterans Administration Hospital, San Diego, CA, USA). The limit of detection for allopregnanolone was $35 \cdot 0 \pm 2 \cdot 5 \mathrm{pg} /$ tube. Intra-assay coefficient was $10 \cdot 9 \%$.

\section{Western blot analysis}

The protein expression of the neurosteroidogenic enzymes, $5 \alpha \mathrm{R} 1$ and $5 \alpha \mathrm{R} 2$, was determined by western blot analyses. Brain tissue was homogenised in RIPA protein extraction buffer $(50 \mathrm{mM}$ Tris- $\mathrm{HCl}, 150 \mathrm{mM} \mathrm{NaCl}, 1 \%$ Nonidet $P-40$, $0 \cdot 5 \%$ sodium deoxycholate, $0 \cdot 1 \%$ SDS with Complete Protease Inhibitor Cocktail (Roche Diagnostics Australia Pty Ltd) and PhosStop Phosphatase Inhibitor Cocktail Protein (Roche Diagnostics)). The protein concentration of each sample was then determined using BCA Protein Assay kit (Pierce, Rockford, IL, USA). Proteins (20 $\mu \mathrm{g}$ total protein) were separated by electrophoresis on precast NuPAGE Novex 12\% Bis/Tris gels (Invitrogen) before transfer to Hybond-P PVDF membrane (GE Healthcare, Sydney, NSW, Australia). Membranes were blocked in BSA Blocking Solution ( $5 \% \mathrm{w} / \mathrm{v}$ BSA, 5\% w/v skim milk in $1 \times$ TBS-T $(25 \mathrm{mM}$ Tris- $\mathrm{HCl}, 15 \mathrm{mM} \mathrm{NaCl}, 0 \cdot 1 \% \mathrm{v} / \mathrm{v}$ Tween-20)) for $1 \mathrm{~h}$ before incubation overnight in primary antibody. Goat polyclonal antibody against $5 \alpha \mathrm{R} 1$ (NB100-1491; Novus Biologicals, Littleton, CO, USA) and goat polyclonal antibody to $5 \alpha \mathrm{R} 2$ (Ab27469; AbCam, Cambridge, UK) were used at a dilution of 1:1000. The immunoreactive protein was detected using the ECL Western Blotting Detection kit (GE Healthcare) and LAS-3000 Imaging System (Fuji Photo Film, Tokyo, Japan) following incubation with anti-goat secondary antibody conjugated to HRP (P0449; DakoCytomation, Glostrup, Denmark) at 1:3000 dilution for $1 \mathrm{~h}$ at room temperature. The relative amount of $5 \alpha \mathrm{R} 1(\sim 26 \mathrm{kDa})$ and $5 \alpha \mathrm{R} 2(\sim 29 \mathrm{kDa})$ protein was quantified using Multigauge v2.4 software (Fuji Photo Film), adjusted to $\beta$-actin loading control (ab8227-50; Abcam) and an internal control sample (human placental protein extract) present on each gel. Control membranes in which the $5 \alpha \mathrm{R} 1$ primary antibody was omitted and where the primary antibody was pre-incubated with the blocking peptide (NB100-1491PEP; Novus Biologicals) confirmed the specificity of the $26 \mathrm{kDa}$ band. For $5 \alpha \mathrm{R} 2$, control membranes in which the primary antibody was omitted and replaced by goat IgG (sc-2028; Santa Cruz Biotechnology, Santa Cruz, CA, USA) confirmed specificity of the $29 \mathrm{kDa}$ band.

\section{Immunohistochemistry}

In all, $8 \mu \mathrm{m}$ coronal sections were cut from paraffinembedded brain tissue blocks using a rotary microtome. Sections were dewaxed in xylene and rehydrated through a graded series of ethanol/water washes. Endogenous peroxidase activity was inhibited by incubation in 3\% hydrogen peroxide in methanol. Antigen recovery was performed with Reveal It Solution (ImmunoSolution Pty Ltd, Everton Park, Qld, Australia) as per the manufacturer's instructions, before being blocked with BSA in PBS (0.1 M PBS, pH $7 \cdot 2$ with $0.5 \% \mathrm{w} / \mathrm{v} \mathrm{BSA}, 0.05 \% \mathrm{w} / \mathrm{v}$ saponin and $0.05 \% \mathrm{v} / \mathrm{v}$ sodium azide). Sections were then incubated in 1:5000 dilution of either glial fibrillary acidic protein (GFAP) (monoclonal anti-GFAP antibody (G3893; Sigma-Aldrich)), myelin basic protein (MBP) (monoclonal anti-MBP antibody (M9434; Sigma-Aldrich)) or activated caspase-3 (anti-h/m caspase-3 active (AF835; R\&D Systems, Minneapolis, MN, USA)) primary antibodies for 3 days. Sections were washed in PBS, followed by incubation in the appropriate biotinylated secondary antibodies: polyclonal rabbit anti-mouse (E0354; DakoCytomation), anti-rat IgG (B7139; Sigma-Aldrich) and anti-rabbit IgG (BA1000; Vector Labs, Burlingame CA, USA) respectively. Sections were then washed and incubated overnight in streptavidin-biotin-HRP complex (RPN1051V; Amersham). Labelling was revealed using 3,3'-diaminobenzidine tetrahydrochloride solution as a chromagen. Sections were mounted with DEPX (Merck) and examined using a Zeiss Axioskop Microscope. Images were acquired using a SPOT RT digital camera (Diagnostic Instruments, Sterling Heights, MI, USA). GFAP, MBP and caspase-3 immunostaining were examined in subcortical white matter and hippocampal CA1; additionally, GFAPstained sections from the dentate region of the hippocampus were also analysed. The GFAP and MBP immunoreactivities were analysed by densitometry using Image 1.40 (National Institutes of Health, Bethesda, MD, USA) and made binary by adjusting the threshold manually, with the per cent area of coverage recorded for four fields of view per region on two sections per animal. Cells positive for activated caspase-3 that showed apoptotic morphology were counted in four fields of view per region (cortex and hippocampal CA1) on two sections per animal. Controls for specificity of primary antibodies were run using the appropriate $\operatorname{IgG}$ substituted for each primary antibody. 


\section{Statistical analysis}

Statistical analysis was carried out using PASW Statistics Package v18.0 for Mac (SPSS, Inc., Chicago, IL, USA). Multiple regression was used to model the measured and continuous outcome variables against the three categorical predictors: finasteride, IUGR and sex, using backwards stepwise mode. Two-way interactions were tested. A two-tailed significance level of 5\% was used throughout. The dependent variables were transformed as required in order to satisfy model assumptions. Subgroup analysis of means was carried out using two-tailed Student's $t$-test where appropriate. Significance levels of $P<0 \cdot 05$ are represented with asterisks in Figs 1-4, where applicable.

\section{Results}

\section{Foetal characteristics}

The mean body and organ weights for foetal guinea pigs are shown in Table 1. A brain to liver weight ratio (BLR) of $>90 \%$ was used to define asymmetric growth restriction in foetuses that received IUGR surgery. Animals that received sham surgeries had BLRs of $\sim 55 \%$, both with and without finasteride treatment.

Regression analyses found that foetal guinea pigs with IUGR had significantly reduced placenta weight $(P<0 \cdot 001)$, liver weight $(P<0 \cdot 001)$ and BLRs $(P<0 \cdot 001)$. Finasteride treatment was not associated with foetal body weight change. Following adjustment for other variables, foetal body weight was significantly reduced in IUGR foetuses $(P<0 \cdot 001)$, with the overall body weight of female foetuses, across all treatment groups, reduced in comparison to male animals $(P=0 \cdot 040)$. Mean foetal brain weight was significantly reduced in IUGR foetuses $(P=0 \cdot 005)$ and a marginally significant reduction in brain weights was observed in female foetuses compared with those in males $(P=0 \cdot 051)$. Finasteride treatment alone was not associated with foetal body weight change. Interestingly, however, there was a significant positive interaction effect $(P=0 \cdot 020)$ between finasteride treatment and IUGR.

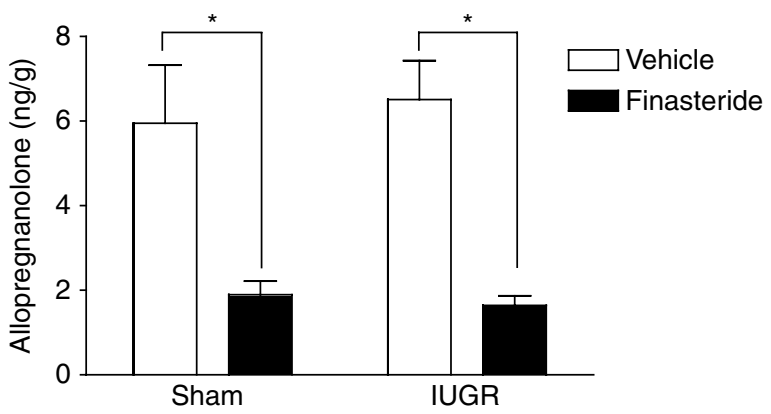

Figure 1 Foetal brain allopregnanolone concentrations with finasteride treatment and IUGR. Relative expression measured by RIA and expressed as mean allopregnanolone (ng/g of wet weight tissue) concentrations \pm S.E.M. $(n=8-10)$. *Significant difference between vehicle- and finasteride-treated animals, $P<0 \cdot 05$ (Student's $t$-test).
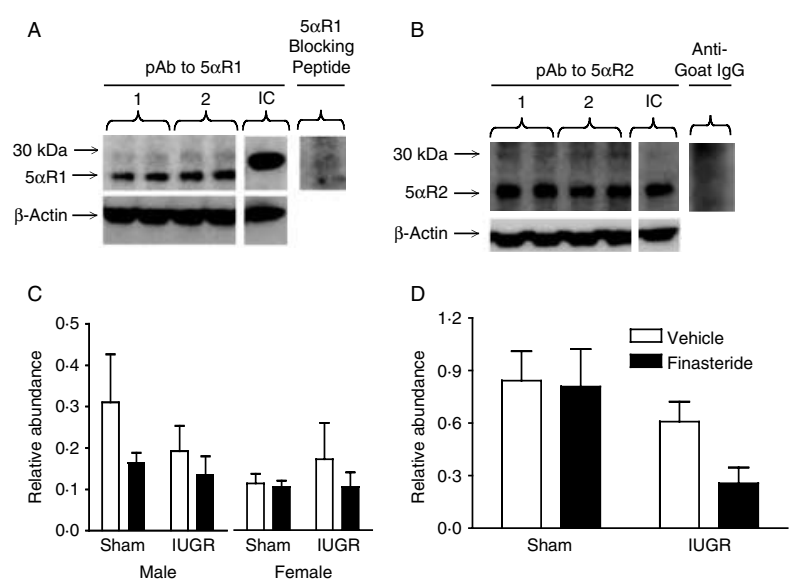

Figure 2 Effect of IUGR and finasteride treatment on the expression of $5 \propto \mathrm{R} 1$ an $5 \propto \mathrm{R} 2$ enzymes in the foetal guinea pig brain.

Representative western blots showing two paired guinea pig brain tissue samples and human placenta internal control $(20 \mu \mathrm{g}$ total protein per lane) and corresponding $\beta$-actin loading controls for $5 \alpha R 1$ (A; $26 \mathrm{kDa}$ band) and 5 $\alpha \mathrm{R} 2$ (B; $29 \mathrm{kDa}$ band). Pre-incubation of primary $5 \alpha \mathrm{R} 1$ antibody with blocking peptide blocked specific binding at $26 \mathrm{kDa}$ in pooled guinea pig brain sample. Goat normal IgG showed no binding at $29 \mathrm{kDa}$ in pooled guinea pig brain sample. The expressions of $5 \alpha \mathrm{R} 1$ for male and female foetuses (C; $n=4-6)$ and $5 \alpha \mathrm{R} 2$ (D; $n=8-10$ ) with IUGR and finasteride treatment are expressed as mean relative abundance \pm S.E.M.

\section{Foetal brain allopregnanolone concentrations}

Figure 1 presents the mean foetal brain concentrations of allopregnanolone with and without IUGR and finasteride treatment. Control animals (sham + vehicle) had average brain allopregnanolone concentrations of $11.63 \pm 2.33 \mathrm{ng} / \mathrm{g}$. Overall, finasteride treatment significantly reduced brain allopregnanolone concentrations $(P=0 \cdot 001)$. Normally grown animals that received finasteride in late gestation had reduced average brain allopregnanolone concentrations of $4 \cdot 20 \pm 0.51 \mathrm{ng} / \mathrm{g}$. IUGR alone did not significantly affect allopregnanolone concentrations in the foetal brain, with a mean allopregnanolone brain concentration of $7 \cdot 72$ $\pm 1.43 \mathrm{ng} / \mathrm{g}$. Brain allopregnanolone was reduced by up to $75 \%$ in IUGR foetuses treated with finasteride (3.09 $\pm 0.57 \mathrm{ng} / \mathrm{g}$ ) compared to control.

\section{Brain $5 \alpha R$ enzyme expression}

The mean abundances of $5 \alpha \mathrm{R} 1$ and $5 \alpha \mathrm{R} 2$ enzymes, expressed relative to internal control and $\beta$-actin loading control, are shown in Fig. $2 \mathrm{C}$ and $\mathrm{D}$ respectively. Representative western blots for both isoforms (Fig. 2A and $\mathrm{B}$ ) with results for negative controls are shown for blots incubated in the presence of $5 \alpha \mathrm{R} 1$ antibody blocking peptide and anti-goat pre-immune serum for $5 \alpha \mathrm{R} 2$, demonstrating specificity of antibody staining. Foetal sex was significantly associated with the expression of the $5 \alpha \mathrm{R} 1$ enzyme isoform $(P=0.008)$, with female foetuses having 

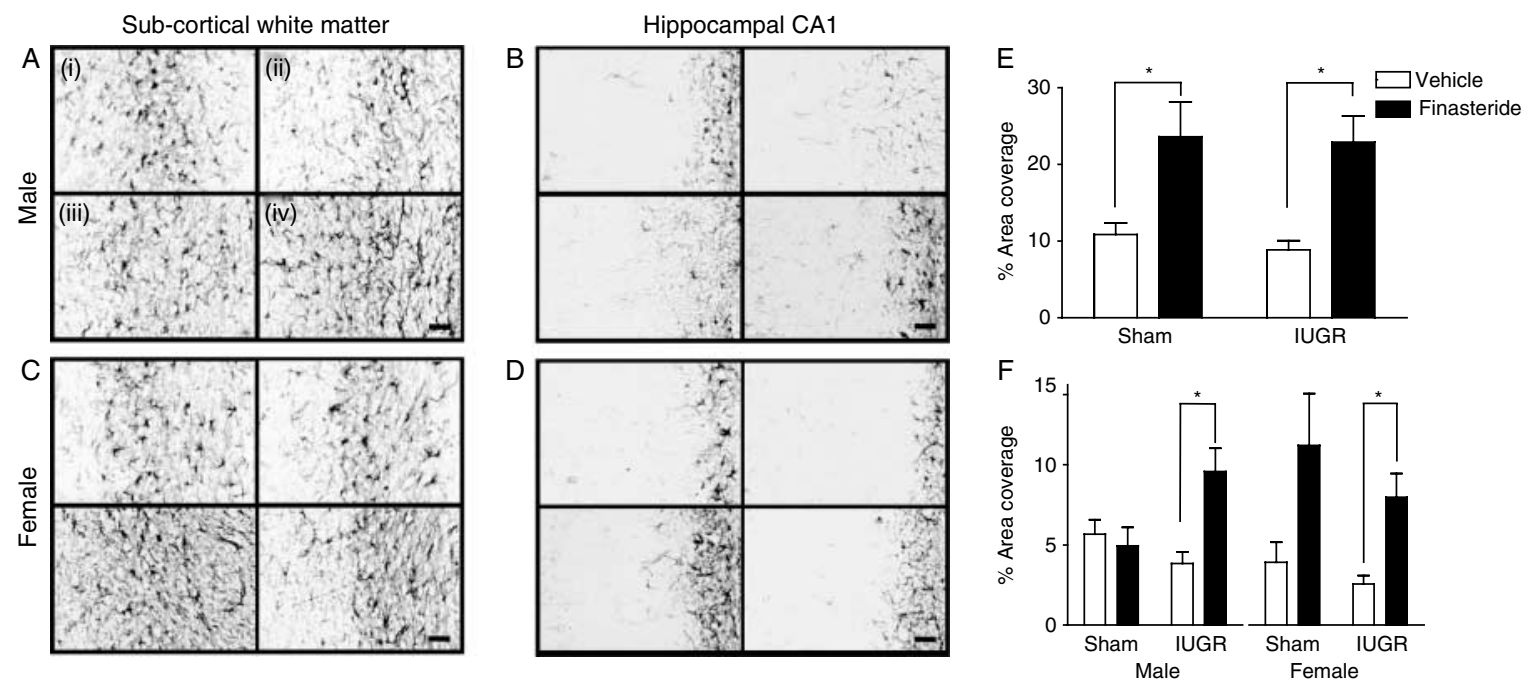

Figure 3 Effect of IUGR and finasteride treatment on glial fibrillary acidic protein (GFAP) immunostaining in the subcortical white matter $(A, C$ and $E)$ and the hippocampal $C A 1$ region $(B, D$ and $F)$. Representative micrographs show staining in male $(A$ and $B)$ and female $(C$ and D) foetal guinea pig brain regions. Staining is expressed as mean percentage coverage of total area \pm s.E.M. (E and F). Foetuses were normally grown, with vehicle (i; sham + veh) or finasteride treatment (iii; sham + fin) and growth restricted with vehicle (ii; IUGR + veh) or finasteride treatment (iv; IUGR + fin). In E $(n=10-12)$ and F ( $n=6-7$ males, $n=5-6$ female foetuses) * indicates significant difference between vehicle and finasteride treatment for male and female foetuses with IUGR $(P<0 \cdot 05$; Student's $t$-test). Horizontal scale bars represent $0 \cdot 1 \mathrm{~mm}$.

lower expression than males. Finasteride and IUGR had no significant effect on the $5 \alpha \mathrm{R} 1$ expression. IUGR was marginally associated with a reduction in the expression of $5 \alpha \mathrm{R} 2$ in the foetal brain $(P=0 \cdot 060$, Fig. $2 \mathrm{~B}$ and $\mathrm{D})$. Finasteride and foetal sex were not associated with changes in the $5 \alpha \mathrm{R} 2$ expression.

\section{GFAP expression}

Figure $3 \mathrm{~A}$ and $\mathrm{C}$ show GFAP immunostaining in the subcortical white matter in male and female foetuses respectively with foetuses that received sham or IUGR surgery and subsequently administered with vehicle or finasteride. The greatest density of GFAP staining appeared to be present in finasteride-treated IUGR male foetuses and finasteride-treated females (Fig. 3A(iii) and C(iii)), however, no significant effect of foetal sex was identified. Animals that were vehicle treated and received either IUGR or sham surgery showed no qualitative increase in GFAP staining in this region. The mean densities of GFAP staining, by proportion of area coverage, for treatment groups with sham and IUGR surgery and vehicle and finasteride treatments are shown in Fig. 3E. Data for the dentate region are not shown.

Regression analysis of GFAP coverage showed that finasteride treatment was associated with a significant increase in GFAP expression in the subcortical white matter $(P<0 \cdot 001)$ and dentate $(P=0 \cdot 009)$ regions. No significant interactions with or effects of IUGR or sex were observed in these regions. This was also confirmed by subgroup analysis of means, which indicated that in both sham and IUGR subgroups finasteride-treated foetuses had a significant increase in GFAP expression (Fig. 3E, $P<0 \cdot 05$ ).

In the CA1 region of the hippocampus, male foetuses with finasteride treatment (Fig. 3B(iv)) and female IUGR foetuses with finasteride treatment (Fig. 3D(iii)) appeared to have the highest degrees of GFAP expression. As an effect of foetal sex was identified (see regression model below), Fig. 3F presents the mean proportion of GFAP staining in the CA1 region divided by treatment groups and foetal sex. Regression analysis of density of GFAP staining in the hippocampal CA1 region found that IUGR was not associated with a change in GFAP expression. A marginally significant increase in overall GFAP expression in this region was observed in female guinea pigs compared with males $(P=0 \cdot 051)$ and while finasteride treatment alone did not have a significant effect on GFAP in the CA1 $(P=0 \cdot 625)$, there was a significant positive interaction between finasteride treatment and $\operatorname{sex}(P=0 \cdot 021)$. A subgroup analysis of means indicated that in the CA1 region, both male and female IUGR foetuses significantly increased GFAP expression compared to IUGR vehicle foetuses of the same sex (Fig. 3F, $P<0 \cdot 05$ ).

\section{$M B P$ expression}

In both the subcortical white matter (Fig. 4A and C) and CA1 region of the hippocampus (Fig. 4B and D), MBP expression was highest in control male (i) and control female foetuses (i). There appeared to be a large reduction in MBP immunostaining in animals that were both growth restricted and 
Sub-cortical white matter
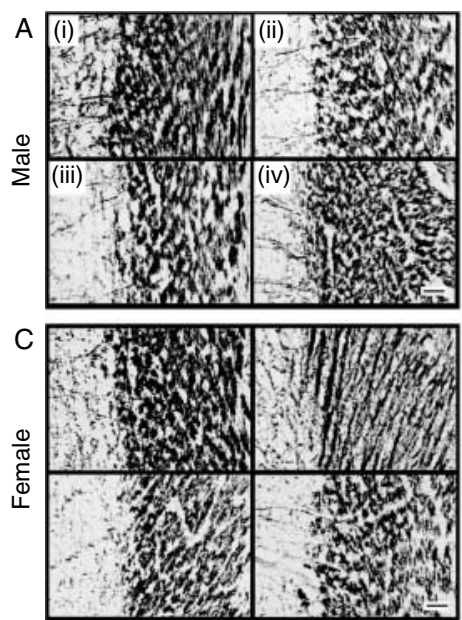

Hippocampal CA1

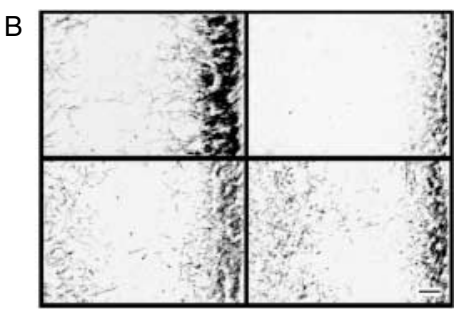

$\mathrm{D}$

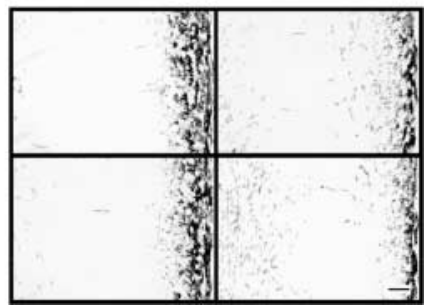

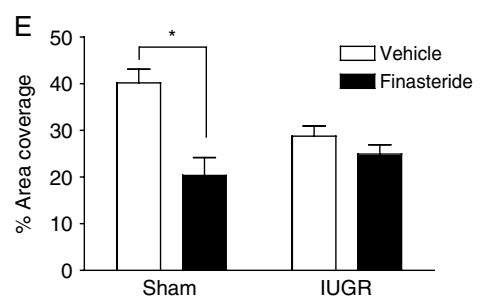

$\mathrm{F}$

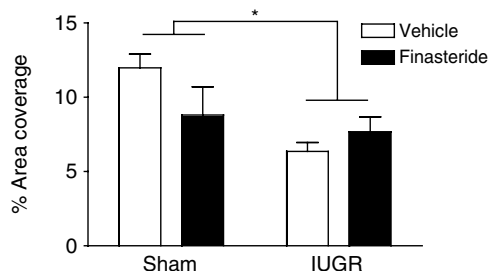

Figure 4 Effect of IUGR and finasteride treatment on myelin basic protein (MBP) immunostaining in the subcortical white matter (A, C and E) and the hippocampal CA1 region (B, D and E). Representative micrographs show staining in male (A and B) and female (C and D) foetal guinea pig brain regions. Staining is expressed as mean percentage coverage of total area \pm s.E.M. (E and F; $n=10-12)$. Foetuses were normally grown, with vehicle (i; sham + veh) or finasteride treatment (iii; sham + fin) and growth restricted with vehicle (ii; IUGR + veh) or finasteride treatment (iv; IUGR + fin). (E) * Indicates significant difference between vehicle- and finasteride-treated sham foetuses.

$(\mathrm{F}) *$ Indicates significant difference between sham and IUGR foetuses $(P<0 \cdot 05$; Student's $t$-test). Horizontal scale bars represent $0 \cdot 1 \mathrm{~mm}$.

received finasteride treatment. The mean per cent coverage of MBP for each treatment group is presented in Fig. $4 \mathrm{E}$ and F.

Multiple regression analysis identified a significant reduction in MBP expression in the subcortical white matter associated with finasteride treatment $(P=0 \cdot 001)$ and IUGR $(P=0 \cdot 003)$. A significant positive interaction between finasteride and IUGR was also identified $(P=0 \cdot 044)$. Additional subgroup analysis of means showed a significant reduction in MBP expression between sham vehicle- and finasteride-treated foetuses (Fig. 4E, $P<0.05$ ) but not in IUGR foetuses with the different drug treatments, indicating a mediation of the effect of finasteride in the presence of IUGR. Foetal sex did not affect MBP expression in this region.

Multiple regression and subgroup analysis identified an association between IUGR and reduced MBP expression in the CA1 region of the hippocampus $(P<0 \cdot 001)$. The different pattern of expression of vehicle and finasteride animals between the sham and IUGR groups (Fig. 4F) suggests an interplay between finasteride treatment and IUGR, which was supported by the regression model showing a significant interaction effect between IUGR and finasteride treatment $(P=0 \cdot 016)$, despite finasteride treatment alone not having a significant effect $(P=0 \cdot 116)$. Foetal sex did not have a significant effect on MBP expression in this region.

\section{Activated caspase-3 expression}

There was no significant effect of finasteride treatment, IUGR or foetal sex on the number of activated caspase-3positive cells in the CA1 region of the hippocampus of the foetal guinea pigs in this study (data not shown).

\section{Discussion}

The neurosteroid allopregnanolone has many associated neuroprotective, developmental and regulatory effects. This study examined late gestation brain development in foetal guinea pigs with the chronic gestational insult, IUGR, in the presence and absence of high late gestation allopregnanolone concentrations. The current model involves chronic placental insufficiency resulting in significantly lower birth weights, with relative brain sparing, typical of IUGR pregnancies. IUGR can be thought of as a chronic adaptation in the foetus that becomes maladaptive, potentially disrupting the neurodevelopmental actions of neurosteroids and leading to strong associations between IUGR, poor neonatal outcome and perinatal brain injury.

In this study, the chronic administration of finasteride during late gestation was successful in markedly reducing allopregnanolone concentrations in the foetal guinea pig brain. It has been proposed that this late gestation reduction in foetal brain allopregnanolone may mimic the change in brain neurosteroid concentration that occurs when a foetus is born preterm and the placenta, as a major source of progesterone, an important precursor of allopregnanolone, is prematurely removed (Hirst et al. 2006). The ability of the preterm infant to synthesise important neurosteroids independently of placentally derived precursors may be limited and the effect of this decline in endogenous steroids on preterm ex utero brain development may influence the vulnerability of the preterm neonatal brain to injury.

Overall, male foetuses were found to have higher levels of expression of $5 \alpha \mathrm{R} 1$ than female foetuses. This may be related to the action of $5 \alpha \mathrm{R} 2$ in androgen and testosterone synthesis 
Table 1 Body and organ weight characteristics of foetal guinea pigs. Values are expressed as mean \pm S.E.M.

\begin{tabular}{|c|c|c|c|c|c|c|}
\hline & $n$ & Body Wgt & Placenta Wgt & Brain Wgt & Liver Wgt & BLR \\
\hline \multicolumn{7}{|l|}{ Control } \\
\hline Female & 6 & $85 \cdot 9 \pm 3 \cdot 3$ & $6 \cdot 3 \pm 0 \cdot 2$ & $2 \cdot 3 \pm 0 \cdot 04$ & $4 \cdot 1 \pm 0 \cdot 3$ & $0.57 \pm 0.04$ \\
\hline Male & 9 & $91 \cdot 1 \pm 2 \cdot 6$ & $6 \cdot 0 \pm 0 \cdot 3$ & $2 \cdot 4 \pm 0 \cdot 02$ & $4 \cdot 6 \pm 0 \cdot 2$ & $0.54 \pm 0.03$ \\
\hline Total & 15 & $89 \cdot 0 \pm 2 \cdot 1$ & $6 \cdot 1 \pm 0 \cdot 2$ & $2 \cdot 4 \pm 0.03$ & $4 \cdot 5 \pm 0 \cdot 2$ & $0.55 \pm 0.03$ \\
\hline \multicolumn{7}{|l|}{ IUGR } \\
\hline Female & 8 & $57 \cdot 9 \pm 4 \cdot 2$ & $4 \cdot 3 \pm 0 \cdot 7$ & $2 \cdot 2 \pm 0 \cdot 03$ & $2 \cdot 5 \pm 0 \cdot 3$ & $1 \cdot 06 \pm 0 \cdot 22$ \\
\hline Male & 8 & $59 \cdot 5 \pm 5 \cdot 5$ & $4 \cdot 4 \pm 0 \cdot 5$ & $2 \cdot 3 \pm 0 \cdot 08$ & $2 \cdot 5 \pm 0 \cdot 3$ & $1 \cdot 03 \pm 0 \cdot 17$ \\
\hline Total & 16 & $58 \cdot 7 \pm 3 \cdot 4$ & $4 \cdot 3 \pm 0 \cdot 4$ & $2 \cdot 3 \pm 0 \cdot 04$ & $2 \cdot 5 \pm 0 \cdot 2$ & $1 \cdot 04 \pm 0 \cdot 13$ \\
\hline \multicolumn{7}{|l|}{ Fin } \\
\hline Female & 4 & $84 \cdot 3 \pm 7 \cdot 0$ & $5 \cdot 0 \pm 0 \cdot 3$ & $2 \cdot 3 \pm 0 \cdot 03$ & $4 \cdot 1 \pm 0 \cdot 6$ & $0.60 \pm 0.08$ \\
\hline Male & 7 & $90 \cdot 4 \pm 3 \cdot 1$ & $6 \cdot 2 \pm 0 \cdot 5$ & $2 \cdot 3 \pm 0 \cdot 04$ & $4 \cdot 6 \pm 0 \cdot 4$ & $0.52 \pm 0.05$ \\
\hline Total & 12 & $87 \cdot 4 \pm 3 \cdot 0$ & $5 \cdot 8 \pm 0 \cdot 4$ & $2 \cdot 3 \pm 0.03$ & $4 \cdot 4 \pm 0 \cdot 3$ & $0.56 \pm 0.04$ \\
\hline \multicolumn{7}{|c|}{ IUGR + Fin } \\
\hline Female & 8 & $53 \cdot 4 \pm 3 \cdot 5$ & $3 \cdot 2 \pm 0 \cdot 5$ & $2 \cdot 0 \pm 0 \cdot 29$ & $2 \cdot 4 \pm 0 \cdot 1$ & $0.95 \pm 0.08$ \\
\hline Male & 3 & $68 \cdot 0 \pm 6 \cdot 4$ & $4 \cdot 1 \pm 0 \cdot 3$ & $2 \cdot 4 \pm 0.08$ & $2 \cdot 8 \pm 0 \cdot 2$ & $0 \cdot 86 \pm 0.04$ \\
\hline Total & 17 & $57 \cdot 1 \pm 2 \cdot 4$ & $3 \cdot 6 \pm 0 \cdot 2$ & $2 \cdot 2 \pm 0 \cdot 12$ & $2 \cdot 5 \pm 0 \cdot 1$ & $0.93 \pm 0.05$ \\
\hline
\end{tabular}

Wgt, weight (g); BLR, brain to liver ratio; control represents sham, vehicle-treated animals; IUGR, intrauterine growth restriction; Fin, finasteride.

as, along with allopregnanolone, $5 \alpha \mathrm{R}$ action is also involved in the synthetic pathways of other steroid hormones. In this study, IUGR did not affect foetal brain concentrations of allopregnanolone. By subgroup analysis, IUGR foetuses had a reduction in the type $25 \alpha \mathrm{R}$ isoform of the enzyme. A previous study carried out by members of our laboratory showed an upregulation of $5 \alpha \mathrm{R} 2$ in the foetal sheep brain following late gestation chronic umbilico-placental embolisation (Nguyen et al. 2003). This may be explained by the different modes of chronic insults used in the two models. Both models showed no change in brain allopregnanolone concentrations despite the changes noted in enzyme expression. Local reductions in allopregnanolone may have been present at the initiation of the chronic-type insults, however, this may not persist to the end of gestation. Acute or transient hypoxic insults during pregnancy have been shown to cause an increase in foetal brain concentrations of allopregnanolone, for a period immediately following the insult (Nguyen et al. 2004). This raises the relative importance of the placenta and other glandular sources for the supply of allopregnanolone and its precursors and the ability for this system to compensate for chronic disruptions of supply, particularly when, as often occurs with IUGR, placental growth or function is compromised. The finding of reduced $5 \alpha \mathrm{R} 2$ expression in the brain of growth-restricted foetuses suggests that IUGR may further limit their neurosteroid synthetic capacity following birth. Such a disruption of neurosteroid synthesis or supply potentially predisposes the vulnerability of the IUGR foetus to neurodevelopmental disorders. The relative importance or otherwise of the various sources of neurosteroids is still unclear and the elucidation of these mechanisms may be useful for the understanding of neurosteroid function in both normal pregnancies and when chronic pathological gestational changes are present.

A key finding of this study is the effect of finasteride treatment and the resultant low concentrations of allopregnanolone on myelination. Correct myelination is essential for the conduction of brain signals and disruption of brain myelination processes during development can lead to lasting neurological, cognitive and motor effects. In the subcortical white matter region, finasteride was associated with significantly reduced myelination and in addition, a significant interaction was also identified between finasteride treatment and IUGR in both regions examined. This interaction indicates that the effect of chronically low foetal brain allopregnanolone concentrations on myelination is altered in foetuses with a chronic perturbation of growth. This is suggestive of a protective mechanism acting in the presence of chronic IUGR, however the mechanism is unclear. These processes may involve the IUGR-induced reduction in $5 \alpha \mathrm{R} 2$ enzyme and consequently a chronic reduction in the conversion of progesterone into other steroids, prior to finasteride treatment. Progesterone has been shown to have positive effects on myelination in the developing CNS, potentially by the enhancement of maturation processes of oligodendrocyte precursors into myelinating oligodendrocytes (Ghoumari et al. 2003, 2005). Therefore, whilst allopregnanolone has been shown to have positive effects on MBP expression, progesterone appears to have a more potent effect. The future investigation of direct progesteroneprogesterone receptor effects in this guinea pig model of IUGR may therefore help to explain the changes observed in foetal brain MBP expression. Together the present observations of changes in MBP expression are consistent with the studies that have previously suggested a trophic effect of neurosteroids on the late gestation myelination (Schumacher et al. 2000) and support the concept that the low neurosteroid levels may negatively impact myelination when endogenous neurosteroid supply is disrupted due to impaired placental function or preterm birth. 
The finding, in this study, that GFAP expression increased when allopregnanolone synthesis was inhibited supports a key regulatory role of this steroid. GFAP is expressed in astrocytes throughout development and when activated by pathological processes (Eng et al. 2000). The increase in GFAP expression may be a consequence of the loss of allopregnanolone-mediated inhibition of neural excitability and hence an increase in excitotoxic cellular processes, cell death and damage. This study identified no significant associations between IUGR and GFAP expression. This is consistent with a previous study that demonstrated no marked difference in GFAP-positive cells in growth-restricted foetal guinea pig brains (Nitsos \& Rees 1990). In vitro studies in organotypic brain slice cultures have previously shown that pretreatment with allopregnanolone reduces astrogliosis following a hypoxic insult (Kruse et al. 2009). Studies of traumatic brain injury in adult rats have also shown that both progesterone and allopregnanolone administration reduces the size of GFAP-positive astrocytes (Djebaili et al. 2005). Despite the increase in GFAP expression when allopregnanolone synthesis was inhibited, qualitative analysis identified no overt areas of glial scarring or obvious signs of damage related to GFAP staining.

We have previously demonstrated the neuroprotective role of allopregnanolone in the foetal sheep brain, when allopregnanolone synthesis was inhibited transiently and acutely. The suppression of allopregnanolone synthesis increases markers of cell proliferation, an effect that is ameliorated when the synthetic neurosteroid, alfaxalone, is used to replace the loss of the endogenous neurosteroid (Yawno et al. 2009). In adult rat models of traumatic brain injury, allopregnanolone administration has also reduced the size of GFAP-positive astrocytes at the site of the experimental lesions (Djebaili et al. 2005), and there is increasing evidence for the effectiveness of allopregnanolone in reducing lesion size and enhancing functional recovery in animal models of traumatic brain injury (Djebaili et al. 2004, 2005, Schumacher et al. 2007). In addition to the previously reported neuroprotective actions of allopregnanolone, this study provides evidence of delayed neurodevelopment in the presence of a low neurosteroid environment and suggests an important role of allopregnanolone in the regulation of developmental processes in the late gestation foetal brain. These findings support the concept that the loss of endogenous neurosteroids and long-term physiological stressors in utero may contribute to the vulnerability of the prematurely born IUGR neonate to abnormal brain development and function. We have also shown that the neurosteroid response of the foetus to an in utero insult is highly dependent on the type and length of insult. The potentially important role of neurosteroids in the regulation of developmental processes and neuroprotection in the foetal brain may provide new directions for the prevention and treatment of neurological disorders that are associated with perinatal insults and pathologies.

\section{Declaration of interest}

The authors declare that there is no conflict of interest that could be perceived as prejudicing the impartiality of the research reported.

\section{Funding}

This work was supported by the National Health and Medical Research Council of Australia (project grant ID\#455527).

\section{References}

de Brito Faturi C, Teixera-Silva F \& Leite JR 2006 The anxiolytic effect of pregnancy in rats is reversed by finasteride. Pharmacology, Biochemistry, and Behavior 85 569-574. (doi:10.1016/j.pbb.2006.10.011)

Compagnone NA \& Mellon SH 2000 Neurosteroids: biosynthesis and function of these novel neuromodulators. Frontiers in Neuroendocrinology 21 1-56. (doi:10.1006/frne.1999.0188)

Di Renzo G, Rosati A, Sarti R, Cruciani L \& Cutuli A 2007 Does fetal sex affect pregnancy outcome? Gender Medicine 4 19-30. (doi:10.1016/S15508579(07)80004-0)

Djebaili M, Hoffman SW \& Stein DG 2004 Allopregnanolone and progesterone decrease cell death and cognitive deficits after a contusion of the rat pre-frontal cortex. Neuroscience 123 349-359. (doi:10.1016/ j.neuroscience.2003.09.023)

Djebaili M, Guo Q, Pettus EH, Hoffman SW \& Stein DG 2005 The neurosteroids progesterone and allopregnanolone reduce cell death, gliosis, and functional deficits after traumatic brain injury in rats. Journal of Neurotrauma 22 106-118. (doi:10.1089/neu.2005.22.106)

Eng L, Ghirnikar R \& Lee Y 2000 Glial fibrillary acidic protein: GFAP-thirty one years (1969-2000). Neurochemical Research 25 1439-1451. (doi:10. 1023/A:1007677003387)

Ghoumari A, Ibanez C, El-Etr M, Leclerc P, Eychenne B, O’Malley B, Baulieu E \& Schumacher M 2003 Progesterone and its metabolites increase myelin basic protein expression in organotypic slice cultures of rat cerebellum. Journal of Neurochemistry 86 848-859. (doi:10.1046/j.14714159.2003.01881.x)

Ghoumari A, Baulieu E \& Schumacher M 2005 Progesterone increases oligodendroglial cell proliferation in rat cerebellar slice cultures. Neuroscience 135 47-58. (doi:10.1016/j.neuroscience.2005.05.023)

Gilbert Evans SE, Ross LE, Sellers EM, Purdy RH \& Romach MK 2005 3 Alpha-reduced neuroactive steroids and their precursors during pregnancy and the postpartum period. Gynecological Endocrinology 21 268-279. (doi:10.1080/09513590500361747)

He J, Hoffman SW \& Stein DG 2004 Allopregnanolone, a progesterone metabolite, enhances behavioral recovery and decreases neuronal loss after traumatic brain injury. Restorative Neurology and Neuroscience 22 19-31.

Hirst JJ, Yawno T, Nguyen P \& Walker DW 2006 Stress in pregnancy activates neurosteroid production in the fetal brain. Neuroendocrinology 84 264-274. (doi:10.1159/000097990)

Kokate TG, Banks MK, Magee T, Yamaguchi S \& Rogawski MA 1999 Finasteride, a 5alpha-reductase inhibitor, blocks the anticonvulsant activity of progesterone in mice. Journal of Pharmacology and Experimental Therapeutics 288 679-684.

Kruse M, Rey M, Barutta J \& Coirini H 2009 Allopregnanolone effects on astrogliosis induced by hypoxia in organotypic cultures of striatum, hippocampus, and neocortex. Brain Research 1303 1-7. (doi:10.1016/ j.brainres.2009.09.078)

Lackman F, Capewell V, Richardson B, daSilva O \& Gagnon R 2001 The risks of spontaneous preterm delivery and perinatal mortality in relation to size at birth according to fetal versus neonatal growth standards. American Journal of Obstetrics and Gynecology 184 946-953. (doi:10.1067/mob.2001.111719)

Larroque B, Bertrais S, Czernichow P \& Leger J 2001 School difficulties in 20-year-olds who were born small for gestational age at term in a regional cohort study. Pediatrics 108 111-115. (doi:10.1542/peds.108.1.111) 
Mallard C, Loeliger M, Copolov D \& Rees S 2000 Reduced number of neurons in the hippocampus and the cerebellum in the postnatal guinea-pig following intrauterine growth-restriction. Neuroscience 100 327-333. (doi:10.1016/S0306-4522(00)00271-2)

McKendry AA, Palliser HK, Yates DM, Walker DW \& Hirst JJ 2010 The effect of betamethasone treatment on neuroactive steroid synthesis in a foetal guinea pig model of growth restriction. Journal of Neuroendocrinology 22 166-174. (doi:10.1111/j.1365-2826.2009.01949.x)

Mitchell B \& Taggart M 2009 Are animal models relevant to key aspects of human parturition? American Journal of Physiology. Regulatory, Integrative and Comparative Physiology 297 R525-R545. (doi:10.1152/ajpregu.00153. 2009)

Nguyen PN, Billiards SS, Walker DW \& Hirst JJ 2003 Changes in 5alphapregnane steroids and neurosteroidogenic enzyme expression in fetal sheep with umbilicoplacental embolization. Pediatric Research 54 840-847. (doi:10.1203/01.PDR.0000088066.47755.36)

Nguyen PN, Yan EB, Castillo-Melendez M, Walker DW \& Hirst JJ 2004 Increased allopregnanolone levels in the fetal sheep brain following umbilical cord occlusion. Journal of Physiology 560 593-602. (doi:10.1113/ jphysiol.2004.069336)

Nicol MB, Hirst JJ, Walker D \& Thorburn GD 1997 Effect of alteration of maternal plasma progesterone concentrations on fetal behavioural state during late gestation. Journal of Endocrinology 152 379-386. (doi:10.1677/ joe.0.1520379)

Nitsos I \& Rees S 1990 The effects of intrauterine growth retardation on the development of neuroglia in fetal guinea pigs. An immunohistochemical and an ultrastructural study. International Journal of Developmental Neuroscience 8 233-244. (doi:10.1016/0736-5748(90)90029-2)

Paul SM \& Purdy RH 1992 Neuroactive steroids. FASEB Journal 6 2311-2322.

Poletti A, Coscarello A, Negri-Cesi P, Colciago A, Celotti F \& Martini L 1998 5 Alpha-reductase isozymes in the central nervous system. Steroids $\mathbf{6 3}$ 246-251. (doi:10.1016/S0039-128X(98)00018-X)
Rees S, Mallard C, Breen S, Stringer M, Cock M \& Harding R 1998 Fetal brain injury following prolonged hypoxemia and placental insufficiency: a review. Comparative Biochemistry and Physiology. Part A, Molecular and Integrative Physiology 119 653-660. (doi:10.1016/S1095-6433(98)01001-0)

Saigal S \& Doyle LW 2008 An overview of mortality and sequelae of preterm birth from infancy to adulthood. Lancet 371 261-269. (doi:10. 1016/S0140-6736(08)60136-1)

Schumacher M, Akwa Y, Guennoun R, Robert F, Labombarda F, Desarnaud F, Robel P, De Nicola AF \& Baulieu EE 2000 Steroid synthesis and metabolism in the nervous system: trophic and protective effects. Journal of Neurocytology 29 307-326. (doi:10.1023/ A:1007152904926)

Schumacher M, Guennoun R, Stein DG \& De Nicola AF 2007 Progesterone: therapeutic opportunities for neuroprotection and myelin repair. Pharmacology \& Therapeutics 116 77-106. (doi:10.1016/j.pharmthera.2007.06.001) Turner AJ \& Trudinger BJ 2009 A modification of the uterine artery restriction technique in the guinea pig fetus produces asymmetrical ultrasound growth. Placenta 30 236-240. (doi:10.1016/j.placenta.2008.11.023)

Yawno T, Yan EB, Walker DW \& Hirst JJ 2007 Inhibition of neurosteroid synthesis increases asphyxia-induced brain injury in the late gestation fetal sheep. Neuroscience 146 1726-1733. (doi:10.1016/j.neuroscience.2007. 03.023)

Yawno T, Hirst JJ, Castillo-Melendez M \& Walker DW 2009 Role of neurosteroids in regulating cell death and proliferation in the late gestation fetal brain. Neuroscience 163 838-847. (doi:10.1016/j.neuroscience.2009. 07.009)

Received in final form 16 November 2010

Accepted 13 December 2010

Made available online as an Accepted Preprint

13 December 2010 